\title{
Fast Continuum Models for Atom Probe Simulation and Reconstruction
}

\author{
Charles Fletcher ${ }^{1 *}$, Michael Moody ${ }^{1}$ and Daniel Haley ${ }^{1}$ \\ 1. Department of Materials, Parks Road, University of Oxford, Oxford, UK \\ * Corresponding author: charles.fletcher@materials.ox.ac.uk
}

The current algorithm of choice for performing atom probe reconstructions is the point projection method. This algorithm makes a number of geometric assumptions regarding the apex shape and field evaporated ion trajectories: assuming ions follow a mathematical projection off a hemispherical cap onto a plane representing the detector [1]. These simplistic assumptions are particularly problematic when considering heterogeneous materials systems, such as semiconductors, where they fail to capture the changes in sample geometry due to microstructure with differing evaporation fields, dielectric behavior, laser anisotropy, and underlying crystallography. These properties influence the trajectories of evaporated ions and introduce distortions into point projection reconstructions [2].

A number of proposals exist for 'correcting' such distortions including novel reconstruction protocols $[3,4,5]$ and the relaxation of conventional reconstruction parameters to allow for spatial or temporal variability $[6,7]$. However, these protocols are either restricted in their application to particular system geometries, are highly parameterized and unconstrained, or are too computationally intensive for current application to experimental datasets. Even following their application significant distortions within reconstructions typically remain: introducing error into calculated local compositions and interface profiles. These proposals also ignore the known phenomenon of trajectory overlap, which introduce errors surrounding interfaces that can propagate throughout reconstructions.

In order to correct for such distortions a new algorithm accounting for the deflection of ion trajectories under the changes in sample geometry is required. We propose a model-driven reconstruction algorithm where a continuum model, initialized with the sample geometry prior to evaporation, is utilized to derive the reconstruction mapping: a time-dependent function relating trajectory start positions on the sample surface to their impact positions on the detector. When inverted such a mapping could be directly applied to performing reconstruction, assuming model parameters are correctly calibrated. The framework allows for additional physical effects, such as dielectric behavior and crystal anisotropy, to be included depending on the knowledge of the simulated material system available.

Our model uses the level set method to track the sample geometry and extends previous curvature flow approximations for field evaporation $[8,9]$ by coupling with an electrostatic solver: either through a Boundary Element Method (BEM), or a FEM-BEM coupling depending on whether semiconductors and insulators are also being modeled (see Figure 1). Such methods also allow for ion trajectory integration without the requirement of an external mesh spanning up to the detector: increasing the efficiency of the solution and reducing the physical memory required. The level set framework is also very flexible, making it straightforward to couple additional physics with the model. One example is crystallographic anisotropy, where generalizing the material evaporation field parameter to some anisotropy function can capture crystallographic faceting of the sample surface. Locally defining different anisotropy functions allows for the simulation of different crystal grains and grain boundaries (see Figure 2). Our continuum model allows for the simulation of the evaporation of complex materials 
systems on the scale of minutes, rather than the hours to days currently required by the state-of-the-art atomic models $[10,11]$, while successfully capturing much of the relevant field evaporation physics.

Currently we are working on extending our continuum model to three dimensions (3D), increasing the efficiency of the reconstruction mapping generation, and exploring new approaches to handling and capturing trajectory overlap information. Such work is a pivotal step towards developing a new aberration correcting reconstruction protocol applicable to full size experimental datasets.

[1] P. Bas et al, Appl Surf Sci. 87/88 (1995), p. 298-304.

[2] D. Melkonyan et al, Ultramicroscopy. 179 (2017), p. 100-107.

[3] D. Beinke, C. Oberdorfer and G. Schmitz, Ultramicroscopy. 165 (2016), p. 34-41.

[4] N. Rolland et al, Microsc Microanal. 23 (2017), p. 247-254.

[5] D. Beinke and G. Schmitz, Microsc Microanal. Epub (2018), p. 1-8.

[6] B. Gault et al, Ultramicroscopy. 111 (2011), p. 1619-1624.

[7] D. J. Larson et al, Journal of Microscopy. 243 (2011), p. 15-30.

[8] D. Haley, M. P. Moody and G. D. W. Smith, Microsc Microanal. 19 (2013), p. 1709-1717.

[9] D. Haley, P. A. J. Bagot and M. P. Moody, Materials Characterization. 146 (2018), p. 299-306.

[10] N. Rolland et al, Microsc Microanal. 21 (2015), p. 1649-1656.

[11] C. Oberdorfer, S. M. Eich and G. Schmitz, Ultramicroscopy. 128 (2013), p. 55-67.
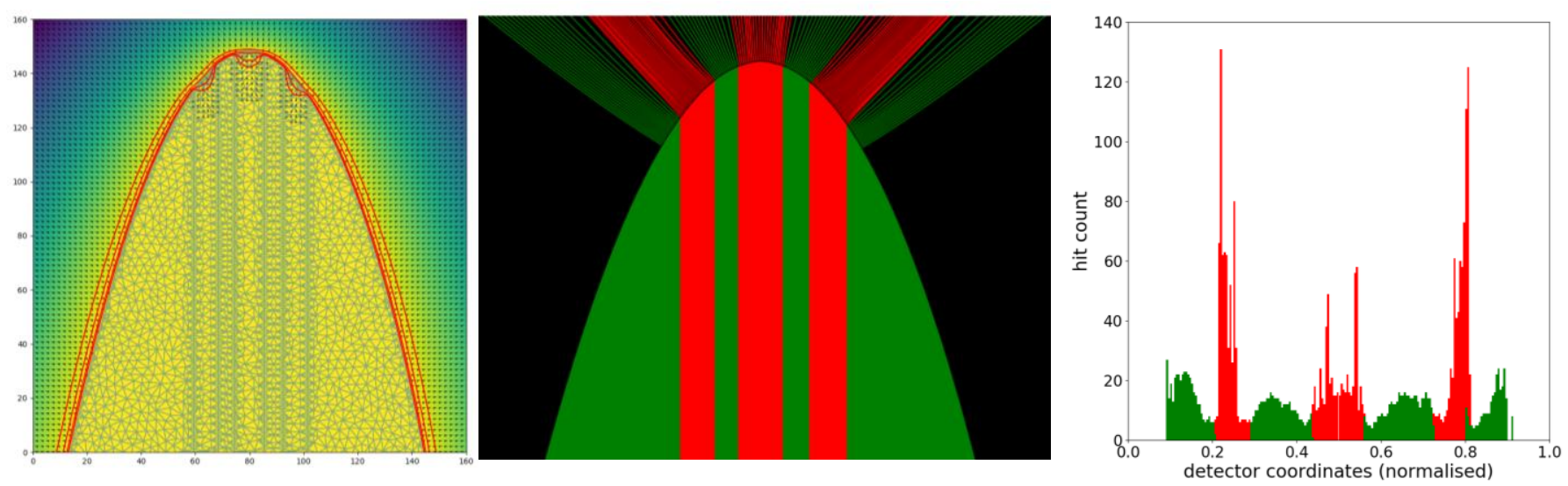

Figure 1. FEM-BEM simulation of vertical dielectric multilayers (a), ion trajectory lensing (b), and the resulting simulated one-dimensional hit-map (c).
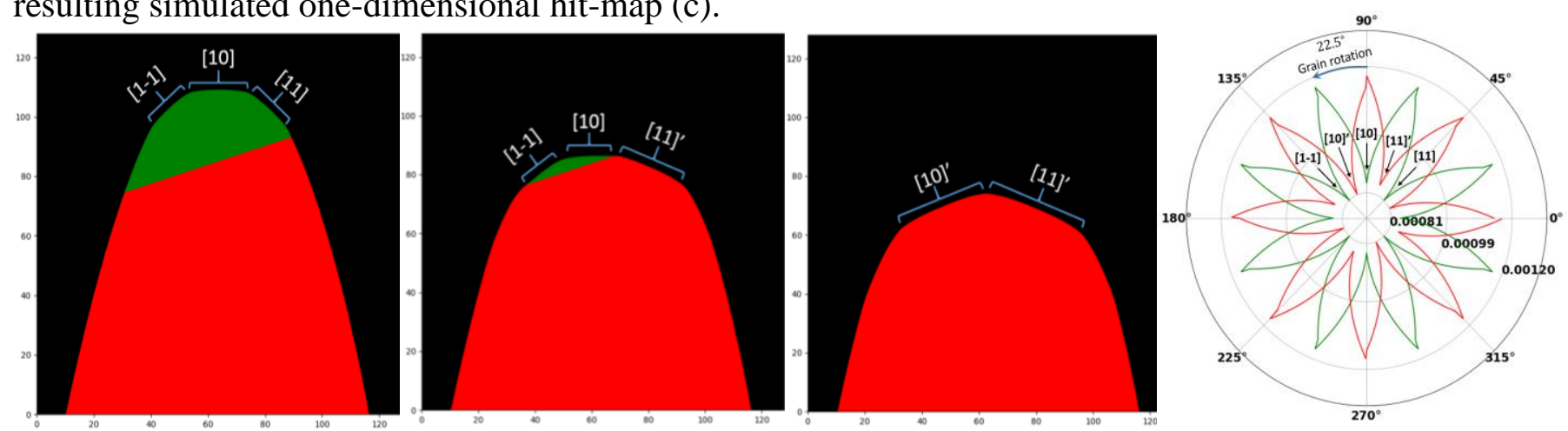

Figure 2. Simulation of a diagonal grain boundary prior to surface intersection (a), during surface intersection (b), and following complete evaporation of the green crystal grain (c). The evaporation anisotropy functions for the two crystal grains are given in $(\mathrm{d})$. 